\title{
Coronary artery pattern and age impact exercise performance late after the arterial switch operation
}

Sara K. Pasquali, MD, Bradley S. Marino, MD, MPP, MSCE, Michael G. McBride, PhD, Gil Wernovsky, MD, and Stephen M. Paridon, MD

From The Cardiac Center at The Children's Hospital of Philadelphia, University of Pennsylvania School of Medicine, Philadelphia, $\mathrm{Pa}$.

Presented in part at the American College of Cardiology 2006 Scientific Sessions, Atlanta, Georgia.

Received for publication March 28, 2007; revisions received May 16, 2007; accepted for publication June 5, 2007.

Address for reprints: Stephen M. Paridon, MD, Associate Professor of Pediatrics, Director of Exercise Physiology Program, The Children's Hospital of Philadelphia, 2nd Floor Main, 34th St. and Civic Center Blvd, Philadelphia, PA 19104 (E-mail: paridon@ email.chop.edu).

J Thorac Cardiovasc Surg 2007;134:1207-12 $0022-5223 / \$ 32.00$

Copyright $(C) 2007$ by The American Association for Thoracic Surgery

doi:10.1016/j.jtcvs.2007.06.022
Objective: The impact of coronary artery pattern on exercise performance after the arterial switch operation is unknown. The purpose of this study was to evaluate the relationship between coronary artery pattern and exercise performance late after the arterial switch operation.

Methods: Patients who underwent the arterial switch operation and were referred for exercise stress testing between January of 1996 and April of 2005 were included. Univariate and multivariate analyses were performed to identify risk factors for lower maximum heart rate and percent of predicted peak oxygen consumption.

Results: Fifty-three patients were included; $72 \%$ were male. The median age at the arterial switch operation was 5 days (1 day to 3.6 years); $32 \%$ had concurrent ventricular septal defect repair. The time from the arterial switch operation to exercise stress testing was 14.1 years (7.7-20.6 years). There were 37 patients with the usual coronary artery pattern, and 16 patients $(30 \%)$ with variant coronary artery patterns. At exercise stress testing, there was no difference in respiratory exchange ratio (1.16 \pm 0.1 for both), indicating similar effort. Compared with patients with the usual coronary artery pattern, patients with variant coronary artery patterns had a significantly lower maximum heart rate $(177 \pm 16$ beats/min [89\% predicted] vs $186 \pm 11$ beats $/$ min [93\% predicted], respectively, $P=.04$ ). Percent of predicted peak oxygen consumption was not significantly different between patients with the usual coronary pattern and patients with variant coronary artery patterns $(89 \% \pm$ $20 \%$ vs $80 \% \pm 17 \%$, respectively, $P=.12$ ). In multivariate analysis, variant coronary artery patterns $(P=.03)$ and ventricular septal defect $(P=.004)$ were predictors of significantly lower maximum heart rate and were associated with a trend toward lower percent of predicted peak oxygen consumption $(P<.09)$. Longer follow-up time was the strongest predictor of lower percent of predicted peak oxygen consumption $(P<.001)$.

Conclusions: Variant coronary artery patterns are associated with chronotropic impairment, and longer follow-up time is the strongest predictor of diminished aerobic capacity late after arterial switch operation.

$\mathrm{T}$ he arterial switch operation (ASO) has become the primary surgical approach used for correction of transposition of the great arteries (TGA). ${ }^{1-4}$ Compared with the Mustard and Senning operations, the ASO restores the left ventricle as the systemic pumping chamber and is associated with improved midterm outcomes. ${ }^{5-7}$

Because of variations in coronary anatomy associated with TGA, and the transfer of the coronary arteries during ASO, much attention has been paid to the relationship between the coronary anatomy pattern and outcome..$^{8-11}$ In a recent metaanalysis, single and intramural coronary patterns were associated with significantly increased all-cause mortality. ${ }^{12}$ It is not known whether coronary artery pattern 

Abbreviations and Acronyms
ASO $=$ arterial switch operation
$\mathrm{EST}=$ exercise stress test
RER $=$ respiratory exchange ratio
TGA $=$ transposition of the great arteries
$\mathrm{VO}_{2}=$ peak oxygen consumption
VSD $=$ ventricular septal defect

impacts morbidity after ASO. The effect of coronary pattern on exercise performance after ASO has not been assessed. It is known that patients with the usual coronary pattern have a normal cardiopulmonary response to exercise after ASO. ${ }^{13}$ However, the potential for sympathetic denervation and alteration in myocardial perfusion as the result of abnormalities in the course of the coronary arteries themselves, or more extensive surgical manipulation necessary for reimplantation, may be greater in those with variant coronary patterns. The purpose of this study was to compare hemodynamic and metabolic parameters during an exercise stress test (EST) in patients with variant and usual coronary artery patterns and to identify other potential risk factors for poor exercise performance late after ASO.

\section{Materials and Methods \\ Patient Population}

Patients who underwent the ASO and were referred for an EST at our institution between January of 1996 and April of 2005 were eligible for inclusion. Those who did not have metabolic measurements performed during EST, had submaximal effort during EST (defined as a maximum respiratory exchange ratio [RER] $<1.10$ or an increase in RER to $<1.40$ during the first 2 minutes of the recovery phase), had an unknown coronary artery pattern, or had a known coronary obstruction were excluded. This study was approved by our institutional review board.

\section{Data Collection}

Patient information was collected from our institutional cardiac databases. Baseline demographics, diagnoses in addition to TGA, and procedures before ASO were obtained from patient charts. Surgical data were collected from operative notes, including weight, age, and concurrent procedures with ASO. Patients' coronary artery patterns were also obtained from the operative record or from the preoperative cardiac catheterization report or clinical record if not delineated in the operative note. Data on reintervention after the ASO were obtained from the patient chart. Echocardiographic data at the time of EST were obtained from our institutional echocardiographic database, including shortening fraction, degree of aortic and pulmonary insufficiency, and stenosis.

\section{Exercise Testing}

Exercise protocol. All subjects exercised to maximal volition using an electronically braked cycle ergometer (Bosch 601). The protocol consisted of 3 minutes of pedaling in an unloaded state followed by a ramp increase in work rate to maximal exercise. The steepness of the ramp protocol was designed to achieve the subjects' predicted work rate in 10 to 12 minutes of cycling time.

Cardiac monitoring. A 12-lead electrocardiogram (Marquette Case-12 Milwaukee, Wis) was obtained at rest in the supine, sitting, and standing positions. A 12-lead electrocardiogram was obtained during each minute of exercise and the first 10 minutes of recovery. Cardiac rhythm was monitored continuously throughout the study.

Metabolic measurements. Metabolic data were obtained throughout the exercise study and for the first 2 minutes of recovery on a breath-by-breath basis using a metabolic cart (Sensor Medics, Yorba Linda, Calif). Parameters measured included minute oxygen consumption, minute carbon dioxide production, minute ventilation, and RER (minute carbon dioxide production/minute oxygen consumption). Ventilatory anaerobic threshold was measured by the V-slope method. Data were compared with healthy age and sexmatched children using a similar exercise protocol as reported by Cooper and colleagues. ${ }^{14}$

\section{Statistical Analysis}

Continuous variables are expressed as mean and standard deviation, or median and range, where appropriate. Univariate analysis using the Student $t$ and Fisher exact tests was performed to compare baseline demographic and surgical data, and echocardiographic and EST data in patients with usual versus variant coronary pattern. The Fisher exact test was also performed to compare the proportion of patients with a maximum heart rate less than $85 \%$ predicted in the usual versus variant coronary pattern groups. In healthy children of all ages performing a bicycle ergometer protocol, the maximum heart rate expected is approximately 200 beats/min with a standard deviation of 7 beats/min. Therefore, patients with a heart rate less than $85 \%$ predicted (or a heart rate of 170 beats/min) have a maximum heart rate more than 3 standard deviations below the predicted normal value. ${ }^{15,16}$ Multivariate analysis was performed using stepwise linear regression to identify predictors of lower maximum heart rate and percent of predicted peak oxygen consumption $\left(\mathrm{VO}_{2}\right)$. All variables that reached statistical significance in the univariate analysis, as well as in the variant coronary pattern, were used in the multivariate analysis. All $P$ values were 2-sided.

\section{Results}

\section{Patient Population}

Sixty-four ASO patients performed ESTs at our institution between January of 1996 and April of 2005. Eleven patients were excluded for the following reasons: submaximal EST $(\mathrm{n}=5)$, no metabolic measurements $(\mathrm{n}=3)$, unknown coronary artery pattern $(\mathrm{n}=2)$, and known coronary obstruction $(\mathrm{n}=1)$. The remaining 53 patients comprise our study cohort.

\section{Coronary Pattern}

The majority of patients $(n=37,70 \%)$ had the usual coronary pattern seen in TGA, where the right coronary artery arises from the right and posterior facing sinus, and the left coronary artery arises from the left and anterior facing sinus before branching into the left anterior descend- 
TABLE 1. Population characteristics

\begin{tabular}{|c|c|c|c|c|}
\hline & \multirow[b]{2}{*}{ Overall $(n=53)$} & \multicolumn{3}{|c|}{ Coronary pattern } \\
\hline & & Usual $(n=37)$ & Variant $(n=16)$ & $P$ value \\
\hline Median age at ASO & $5 d(1 d-3.6 y)$ & $5 d(1 d-3.6 y)$ & $5 d(3-14 d)$ & NS \\
\hline Male & $38(72 \%)$ & $28(76 \%)$ & $10(63 \%)$ & NS \\
\hline VSD & $18(34 \%)$ & $11(30 \%)$ & $7(44 \%)$ & NS \\
\hline Balloon atrial septostomy & $39(74 \%)$ & $28(76 \%)$ & $11(69 \%)$ & NS \\
\hline Pulmonary artery band & $2(4 \%)$ & $1(3 \%)$ & $1(1 \%)$ & NS \\
\hline Concurrent surgery w/ASO & $20(37 \%)$ & $12(32 \%)$ & $8(50 \%)$ & NS \\
\hline Reintervention & $23(43 \%)$ & $12(32 \%)$ & $11(68 \%)$ & .02 \\
\hline
\end{tabular}

$V S D$, Ventricular septal defect; $A S O$, arterial switch operation; $N S$, not significant.

ing and left circumflex coronary arteries. ${ }^{17}$ The remaining 16 patients $(30 \%)$ had variant coronary patterns: circumflex from right coronary artery $(n=6)$, inverted right coronary artery and circumflex $(n=1)$, and single and/or intramural coronary artery $(\mathrm{n}=9)$.

\section{Population Characteristics}

Baseline data for the entire cohort and for those with the usual and variant coronary patterns are shown in Table 1 . The majority of patients were male, underwent the ASO in the neonatal period, and had a balloon atrial septostomy performed before surgery. One patient with TGA and ventricular septal defect (VSD) who underwent balloon atrial septostomy as a neonate did not undergo the ASO until age 3 years. At the time of ASO, 37\% of patients underwent concurrent procedures. In the group with the usual coronary pattern, 12 patients (32\%) underwent concurrent procedures, including VSD closure $(\mathrm{n}=11)$ and pulmonary artery band takedown $(\mathrm{n}=1)$. In the variant coronary artery pattern group, 8 patients $(50 \%)$ underwent 15 concurrent procedures: VSD closure $(\mathrm{n}=6)$, arch augmentation $(\mathrm{n}=$ $3)$, pulmonary artery augmentation $(\mathrm{n}=3)$, Aubert procedure $(n=2)$, and pulmonary artery band takedown $(n=1)$.

There was no statistical difference between those with usual versus variant coronary artery patterns for any of the baseline characteristics listed in Table 1, with the exception of reintervention after ASO. In the group with the usual coronary artery pattern, 12 patients (32\%) underwent 17

TABLE 2. Baseline data at the time of exercise stress test

\begin{tabular}{lcc}
\hline & \multicolumn{2}{c}{ Coronary pattern } \\
\cline { 2 - 3 } & Usual $(\mathbf{n}=\mathbf{3 7})$ & Variant $(\mathbf{n}=\mathbf{1 6})^{*}$ \\
\hline Follow-up time & $14.1 \pm 3.1 \mathrm{y}$ & $13.5 \pm 4.0 \mathrm{y}$ \\
Age at EST & $14.2 \pm 2.9 \mathrm{y}$ & $13.5 \pm 3.9 \mathrm{y}$ \\
Weight & $54.6 \pm 17.1 \mathrm{~kg}$ & $48.8 \pm 14.3 \mathrm{~kg}$ \\
BMI & $20.4 \pm 3.7$ & $20.4 \pm 3.0$ \\
Resting heart rate & $74 \pm 12$ & $75 \pm 8$
\end{tabular}

$\overline{E S T \text {, Exercise stress test; } B M I \text {, body mass index. }{ }^{*} \text { All } P=\text { not significant }}$ versus usual coronary pattern. reinterventions, including reoperation or catheter-based procedure for pulmonary stenosis $(n=11)$, surgical relief of supravalvar aortic stenosis or arch obstruction $(\mathrm{n}=3)$, atrial septal defect closure $(\mathrm{n}=2)$, and coiling of a bronchial collateral $(\mathrm{n}=1)$. In the group with variant coronary artery patterns, 11 patients (68\%) underwent 16 reinterventions ( $P=.02$ vs usual coronary pattern group), including reoperation or catheter-based procedure for pulmonary stenosis $(n=9)$, surgical relief of supravalvar aortic stenosis or arch obstruction $(\mathrm{n}=3)$, and atrial septal defect closure $(\mathrm{n}=$ 2 ). No patient in either group underwent coronary arteryrelated reintervention.

\section{Baseline Data at Exercise Stress Test}

Baseline data at the time of EST are displayed in Table 2. The median time from ASO to EST was 14.1 years (range 7.7-10.6 years) and was similar between those with usual versus variant coronary artery patterns. There was also no difference between coronary groups in age at EST, body habitus, or resting heart rate (Table 2).

TABLE 3. Predictors of exercise performance

\begin{tabular}{llc}
\hline & \multicolumn{2}{c}{$\boldsymbol{P}$ value } \\
\cline { 2 - 3 } & Univariate & Multivariate \\
\hline Lower maximum heart rate & & \\
VSD & .009 & .004 \\
Variant coronary pattern & .04 & .03 \\
Lower raw V0 & .001 & \\
Lower percent predicted $\mathrm{VO}_{2}$ & .004 & \\
Concurrent procedure $\mathrm{W} / \mathrm{ASO}$ & .02 & \\
Lower percent predicted $\mathrm{VO}_{2}$ & & .001 \\
Longer follow-up time & .001 & .05 \\
VSD & .03 & \\
Variant coronary pattern & .1 & \\
Lower maximum heart rate & .004 & \\
Concurrent procedure w/ASO & .007 &
\end{tabular}

VSD, Ventricular septal defect; $A S O$, arterial switch operation; $V O_{2}$, peak oxygen consumption. 


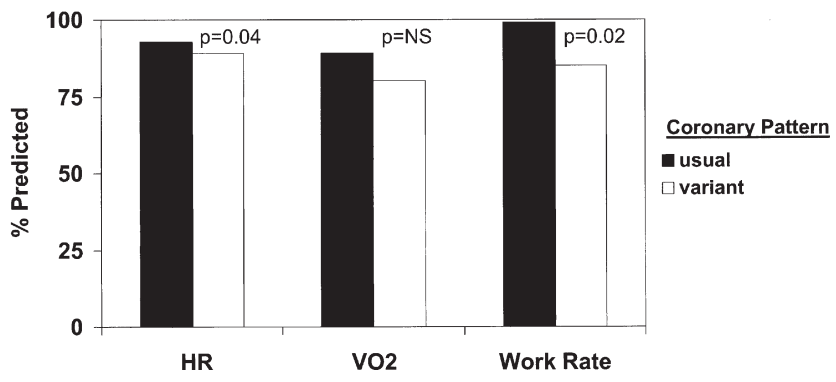

Figure 1. Peak exercise data. $H R$, Heart rate; $\mathrm{VO}_{2}$, oxygen consumption; NS, not significant.

\section{Echocardiographic Data at Exercise Stress Test}

Echocardiographic data were available in 24 patients with the usual coronary artery pattern and in 10 patients with variant coronary patterns (total $n=34 / 5364 \%$ ). The 19 patients without echocardiographic data were primarily followed at other institutions. The median time from EST to echocardiogram was 2 months (range 0-30 months), with $71 \%$ of the echocardiograms performed within 1 year of EST. Shortening fraction was similar in both those with usual and variant coronary patterns $(36 \% \pm 4 \%$ vs $34 \% \pm$ $4 \%)$. One patient in both the usual and variant coronary pattern groups had moderate or more aortic insufficiency ( $4 \%$ vs $10 \%)$. Four patients with the usual coronary pattern had moderate or more pulmonary insufficiency compared with 1 patient in the variant pattern group (17\% vs 10\%). No patients in the usual coronary group had aortic stenosis. One patient in the variant coronary group had aortic stenosis with a peak gradient of $40 \mathrm{~mm} \mathrm{Hg}$. Ten patients (42\%) in the usual coronary pattern group had pulmonary stenosis (mean gradient $32 \pm 11 \mathrm{~mm}$ $\mathrm{Hg}$ ) compared with 5 patients $(50 \%)$ in the variant coronary group (mean gradient $40 \pm 11 \mathrm{~mm} \mathrm{Hg}$ ).

\section{Peak Exercise Data at Exercise Stress Test}

Peak exercise data at the time of EST in those with the usual and variant coronary patterns are shown in Figure 1. There was no difference in RER between the 2 groups $(1.16 \pm 0.1$ for both), indicating similar effort. Compared with patients with the usual coronary artery pattern, patients with variant coronary artery patterns had a significantly lower mean maximum heart rate $(177 \pm 16$ beats/min [89\% predicted] vs $186 \pm 11$ beats $/$ min [ $93 \%$ predicted], $P=.04$ ). In the variant coronary group, 6 of 16 patients $(38 \%)$ had a maximum heart rate that was less than $85 \%$ predicted versus 4 of 37 patients $(11 \%, P=.05)$ in the usual coronary group. Mean maximum work rate was also lower in patients with a variant coronary artery pattern $(2.5 \pm 0.6$ watts $/ \mathrm{kg}[85 \% \pm$ $18 \%$ predicted] vs $3.0 \pm 0.6$ watts $/ \mathrm{kg}[99 \% \pm 24 \%$ predicted], $P=.02$ ). Although raw $\mathrm{VO}_{2}$ was lower in those with a variant coronary artery pattern $(33 \pm 8 \mathrm{~mL} / \mathrm{kg} / \mathrm{min}$ vs
$38 \pm 9 \mathrm{~mL} / \mathrm{kg} / \mathrm{min}(P=.03)$, percent predicted $\mathrm{VO}_{2}$ was not significantly different between the 2 groups $(80 \% \pm$ $17 \%$ vs $89 \% \pm 20 \%, P=.12$ ).

\section{Predictors of Exercise Performance}

Predictors of lower maximum heart rate and percent predicted $\mathrm{VO}_{2}$ are shown in (Table 3),. In multivariate analysis, variant coronary artery pattern $(P=.03)$ and $\operatorname{VSD}(P=$ $.004)$ were independent predictors of significantly lower maximum heart rate. Longer follow-up was the strongest predictor of lower percent predicted $\mathrm{VO}_{2}(P<.001)$ in multivariate analysis. The presence of a $\operatorname{VSD}(P=.05)$ and variant coronary pattern were associated with trends toward lower percent predicted $\mathrm{VO}_{2}(P=.09)$.

\section{Ischemia With Exercise}

Three patients had ST changes noted on the electrocardiogram during their EST. Two patients had a variant coronary pattern, 1 patient had a subsequent normal coronary angiogram, and 1 patient is scheduled for cardiac magnetic resonance imaging to evaluate the coronary arteries. The third patient with ST changes during EST had the usual coronary pattern and a normal coronary angiogram.

\section{Discussion}

This study demonstrates that variant coronary artery patterns in patients with TGA are associated with chronotropic impairment late after ASO. The presence of a VSD was also associated with chronotropic impairment in this population. Longer follow-up time was the strongest predictor of impaired aerobic capacity after ASO.

The ASO is associated with low mortality and improved midterm outcomes compared with atrial level repairs for TGA. $^{5-7}$ Attention has now turned to evaluation of longterm outcomes after ASO. Given the variations in coronary anatomy associated with TGA, and the transfer of the coronary arteries during ASO, several studies have evaluated the relationship between the coronary anatomy pattern and outcome, with mixed results..$^{8-11}$ In a recent meta-analysis, single and intramural coronary patterns were associated with significantly increased all-cause mortality. ${ }^{12}$

This study evaluated the impact of coronary pattern on morbidity late after ASO, specifically on exercise performance. We demonstrated that variant coronary patterns were associated with chronotropic impairment late after ASO. Previous studies have shown that a subset of patients with the usual coronary pattern have chronotropic impairment, but variant coronary patterns were not evaluated. ${ }^{13}$ Similarly, patients with other types of congenital heart defects, such as tetralogy of Fallot, who require significant great artery surgery, or cardiac transplant recipients, who frequently require transection of the great arteries as a part 
of their repair, show evidence of postoperative chronotropic impairment with exercise. ${ }^{18,19}$

In the TGA population, Kondo and colleagues ${ }^{20}$ demonstrated sympathetic denervation, followed by variable degrees of reinnervation in TGA patients following ASO. The degree of reinnervation correlated with chronotropic response to exercise. The impact of variant coronary pattern was not analyzed in this study; however, it is possible that greater dissection is needed during surgery to mobilize and transfer variant coronary arteries, which may impact the degree of denervation and reinnervation postoperatively. Alternatively, the sympathetic innervation pattern itself may be altered in patients with variant coronary patterns.

It is unclear whether the chronotropic impairment in patients with a variant coronary pattern in our study significantly affects their quality of life at present. However, it may be a poor prognostic indicator as we follow these patients into adulthood. A recent study of adult patients with congenital heart disease found that an abnormal heart rate response to exercise was associated with increased mortality. ${ }^{21}$ Thus, close follow-up and further investigation of the impact of chronotropic impairment in patients with TGA and a variant coronary pattern is warranted.

The presence of a VSD was also a significant predictor of chronotropic impairment in multivariate analysis. This has also been shown in patients after isolated repair of a VSD, the reason for which is unclear. ${ }^{22}$ It should be noted that in our study, patients with variant coronary patterns underwent a greater variety of concurrent procedures performed at the time of ASO and reinterventions after ASO. Concurrent surgery at the time of ASO was a predictor of chronotropic impairment in univariate analysis, but it was not significantly associated with heart rate in multivariate analysis. None of the reinterventions performed after ASO were coronary-artery related, and reintervention was not associated with a lower maximum heart rate in univariate or multivariate analysis.

Variant coronary patterns and VSD were also associated with a trend toward lower maximal oxygen consumption in our study. However, the strongest predictor of lower $\mathrm{VO}_{2}$ was a longer duration of follow-up. Follow-up time was not found to be associated with $\mathrm{VO}_{2}$ in a previous study of patients with TGA after ASO; however, the duration of follow-up was shorter in this study. ${ }^{13}$ There are several possible reasons for the association of lower $\mathrm{VO}_{2}$ with longer follow-up time. Because the majority of patients undergo ASO early in the neonatal period, follow-up time is directly related to the era of surgery. It is possible that undergoing ASO early on before refinements in cardiopulmonary bypass and myocardial protection techniques had a negative impact on cardiac function, manifesting as poor exercise performance over the long term. However, only 1 patient in our study population had a shortening fraction less than $28 \%$; the rest had a shortening fraction within normal limits. Thus, it may be that the length of follow-up after ASO, rather than the time of ASO itself, is more strongly related to exercise capacity. There are several reasons for this. It is known that patients with TGA have an abnormal coronary flow reserve after ASO, usually without signs of frank ischemia. ${ }^{23}$ It is possible that these subtle abnormalities in coronary flow may alter the balance between myocardial oxygen supply and demand, such that exercise capacity is reduced. Alternatively, the association of longer follow-up with lower $\mathrm{VO}_{2}$ in patients who undergo the ASO may be due to physical deconditioning. A recent study evaluated the physical activity patterns of 50 children 7 to 14 years after the ASO and found that these patients engaged in significantly less moderate and vigorous physical activity than those who had undergone repair of an atrial defect or VSD, compared with age-matched healthy children. ${ }^{24}$ Although clinic letters did not indicate any restriction to activity, the baseline activity of the patient population in our study was not assessed. Because physical inactivity is known to be associated with poor cardiovascular health, we believe it is of utmost importance to emphasize regular physical activity in the population who undergo the ASO, given that the long-term function of their coronary arteries remains unknown.

\section{Limitations}

This study was a retrospective review of patients who underwent the ASO and were referred for EST at a single institution, and it may be subject to selection bias. Patients in this study are likely to be at the most complex end of the clinical spectrum, as evidenced by the frequency of reintervention, and results may not be applicable to all patients who undergo the ASO. Not all patients had echocardiographic data available at the time of EST, and baseline activity levels and patient quality of life were not assessed in this study.

\section{Conclusions}

A variant coronary artery pattern in patients with TGA is associated with chronotropic impairment late after ASO. Close follow-up of these patients and further investigation into the mechanism and impact of chronotropic impairment in this population are necessary, because an abnormal heart rate response to exercise is known to be associated with increased mortality in adults with congenital heart disease. Longer follow-up time was the strongest predictor of diminished aerobic capacity in the overall cohort, in the absence of significant residual cardiac disease. This suggests a greater emphasis on physical activity and conditioning may be necessary as we follow patients with TGA into adulthood. 


\section{References}

1. Bove EL, Beekman RH, Snider AR, et al. Arterial repair for transposition of the great arteries and large ventricular septal defects in early infancy. Circulation. 1988;78:26-31.

2. Serraf A, Lacour-Gayet F, Bruniaux J, et al. Anatomic correction of transposition of the great arteries in neonates. $J$ Am Coll Cardiol. 1993;22:193-200.

3. Brawn WJ, Mee RBB. Early results for anatomic correction of transposition of the great arteries and for double outlet right ventricle with subpulmonary ventricular septal defect. J Thorac Cardiovasc Surg. 1988;95:230-8

4. Jatene AD, Fontes VF, Paulista PP, et al. Anatomic correction of transposition of the great vessels. J Thorac Cardiovasc Surg. 1976; 72:364-70.

5. Wernovsky G, Hougen T, Walsh E, et al. Midterm results after the arterial switch operation for transposition of the great arteries with intact ventricular septum: clinical, hemodynamic, echocardiographic, and electrophysiologic data. Circulation. 1988;77:1333-44.

6. Colan SD, Boutin C, Castaneda AR, et al. Status of the left ventricle after arterial switch operation for transposition of the great arteries: hemodynamic and echocardiographic evaluation. J Thorac Cardiovasc Surg. 1995;109:311-21.

7. Rhodes LA, Wernovsky G, Keane JF, et al. Arrhythmias and intracardiac conduction after the arterial switch operation. $J$ Thorac Cardiovasc Surg. 1995;109:303-10.

8. Tanel RE, Wernovsky G, Landzberg MJ, Perry SB, Burke RP. Coronary artery abnormalities detected at cardiac catheterization following the arterial switch operation for transposition of the great arteries. Am J Cardiol. 1995;76:153-7.

9. Bonnet D, Bonhoeffer P, Piechaud JF, et al. Long-term fate of the coronary arteries after the arterial switch operation in newborns with transposition of the great arteries. Heart. 1996;76:274-9.

10. Legendre A, Losay J, Touchot-Kone A, et al. Coronary events after arterial switch operation for transposition of the great arteries. Circulation. 2003;108[suppl II]:II-186-90.

11. Bonhoeffer P, Bonnet D, Piechaud JF, et al. Coronary artery obstruction after the arterial switch operation for transposition of the great arteries in newborns. J Am Coll Cardiol. 1997;29:202-6.
12. Pasquali SK, Hasselblad V, Li JS, et al. Coronary artery pattern and outcome of arterial switch operation for transposition of the great arteries: a meta-analysis. Circulation. 2002;106:2575-80.

13. Mahle WT, McBride MG, Paridon SM. Exercise performance after the arterial switch operation for d-transposition of the great arteries. Am J Cardiol. 2001;87:753-8.

14. Cooper DM, Weile-Ravell D, Whipp BJ, Wasserman K. Aerobic parameters of exercise as a function of body size during growth in children. J Appl Physiol Respir Environ Exerc Physiol. 1984;56:628-34.

15. Rowland TW. Aerobic exercise testing protocols. In: Rowland TW, ed. Pediatric Laboratory Exercise Testing: Clinical Guidelines. Champaign, IL: Human Kinetics Publishers; 1993:34.

16. Washington RL, van Gundy JC, Cohen C, et al. Normal aerobic and anaerobic exercise data for North American school-age children. $J \mathrm{Pe}$ diatr. 1988;112:223-33.

17. Yacoub MH, Radley-Smith R. Anatomy of the coronary arteries in transposition of the great arteries and methods for their transfer in anatomical correction. Thorax. 1978;33:418-24.

18. Pastore E, Turchetta A, Attias L, et al. Cardiorespiratory functional assessment after pediatric heart transplantation. Pediatr. Transplant 2001;5:425-9.

19. Mahle WT, McBride MG, Paridon SM. Exercise performance in tetralogy of Fallot: the impact of primary complete repair in infancy. Pediatr Cardiol. 2002;23:224-9.

20. Kondo C, Nakazawa M, Momma K, Kusakabe K. Sympathetic denervation and reinnervation after arterial switch operation for complete transposition. Circulation. 1998;97:2414-9.

21. Diller G, Dimopoulos K, Okonko D, et al. Heart rate response during exercise predicts survival in adults with congenital heart disease. $J$ Am Coll Cardiol. 2006;48:1250-6.

22. Perrault H, Drblik SP, Montiqny M, et al. Comparison of cardiovascular adjustments to exercise in adolescents 8 to 15 years of age after correction of tetralogy of Fallot, ventricular septal defect or atrial septal defect. Am J Cardiol. 1989;64:213-7.

23. Bengel FM, Hauser M, Duvernoy CS. Myocardial blood flow and coronary flow reserve late after anatomical correction of transposition of the great arteries. J Am Coll Cardiol. 1998;32:1955-61.

24. Massin MM, Hovels-Gurich HH, Gerard P, Seghaye MC. Physical activity patterns of children after neonatal arterial switch operation. Ann Thorac Surg. 2006;81:665-70. 